\title{
Change in Semigraph Energy Due to Edge Deletion and its Relation with Distance Energy
}

\author{
Hanumesha. A. G, Meenakshi. K
}

\begin{abstract}
If there is an adjacency matrix A, the sum total of the singular values of $A$ is known as the graph energy. We can find the change in energy of a graph by removing the edges using the inequality theorem on singular values. In this paper we discuss about the change in semigraph energy due to deletion of edges and its relation with distance energy.
\end{abstract}

Index Terms-Semigraph, Adjacency matrix, Energy of graph, Energy of Semigraph, Distance energy.

\section{INTRODUCTION}

Semigraphs, the generalization of graphs was introduced by E.Sampathkumar. Using Semigraphs, we can connect many points by an edge and because of this fact, they have more uses than graphs.. In the second section, we study some preliminary definitions on semigraphs and singular value inequality for a matrix sum. In the third section, we study energy of graph, distance energy of semigraph, energy of a semigraph and then discuss the theorems on semigraph energy change due to the deletion of an edge and its relation with distance energy.

We have a look at the basics of semigraphs in the following section.

\section{A Semigraph}

An ordered pair $(V, X)$ in which $\mathrm{V}$ is a nonempty set of elements called as vertices and the set of $n$ tuples, known as edges is the set $\mathrm{X}$, of distinct vertices, obeying the following conditions where the minimum of $n$ is 2 , is a semigraph.

i)If there are two edges then they have maximum one vertex in common ii) Two elements of the edge set are equal $\left(\mathrm{w}_{1}, \mathrm{w}_{2}, \ldots . \mathrm{w}_{\mathrm{n}}\right)$ and $\left(\mathrm{v}_{1}, \mathrm{v}_{2}, \ldots . \mathrm{v}_{\mathrm{m}}\right)$ are equal if a) $\mathrm{m}=\mathrm{n}$ and $\left.\mathrm{b}\right)$ either $\mathrm{w}_{\mathrm{i}}=\mathrm{v}_{\mathrm{i}}$ for $\mathrm{i}=1$ to $\mathrm{n}$ or $\mathrm{w}_{\mathrm{i}}=\mathrm{v}_{\mathrm{n}+1-\mathrm{i}}$ for $\mathrm{i}=1$ to $\mathrm{n}$. For the edge $\mathrm{E}=\left(\mathrm{u}_{1}, \mathrm{u}_{2}, \ldots . \mathrm{u}_{\mathrm{n}}\right) \mathrm{w}_{1}$ and $\mathrm{w}_{\mathrm{n}}$ are the end vertices and are the $\mathrm{w}_{2}, \ldots \mathrm{w}_{\mathrm{n}-1}$ are the middle end vertices of an edge.

\section{B.Adjacent vertices}

If there are two vertices in a semigraph and if they belong to the same edge then they are known as adjacent and also if they are consecutive then they are adjacent consecutively.

\section{C.Cardinality}

The number of elements of the vertex set lying on that edge is called as the cardinality of an edge.

\section{D.Adjacent edges}

If there is a vertex common in two edges, then the edges are said to be adjacent.

\section{E.Edge Partial}

An edge is said to be edge partial if it is a $1+\mathrm{k}-\mathrm{j}$ tuple $\left(\mathrm{v}_{\mathrm{ij}}, \mathrm{v}_{\mathrm{ij}+1}, \ldots \mathrm{v}_{\mathrm{ik}}\right)$ where $1 \leq j<k \leq n$

\section{F.Edge full}

Any edge under consideration is said to be edge full of a semigraph.

\section{G.Edge partial/Edge full}

Any edge which is either an edge partial or an edge full is said to be edge partial or edge partial.

\section{H.Regular semigraph}

If all the vertices of a semigraph are of same degree, then it is said to be regular.

In this section, we discuss singular value inequality and some definitions on energy of graph and semigraphs and then discuss the theorems on change in energy of semigraph due to the deletion of an edge and its relation with energy distance.

A.Matrix sum inequality with respect to the singular values

Let the $\mathrm{n}$ by $\mathrm{n}$ complex matrix be $\mathrm{X}$ and let us denote its singular values by

$s_{1}(X) \geq s_{2}(X) \geq s_{3}(X) \geq \ldots \ldots \ldots . \geq s_{n}(X) \geq 0$. Let there be only real eignevalues in $X$, that is $\lambda_{1}(X) \geq \lambda_{2}(X) \geq \ldots \geq \lambda_{n}(X)$. Consider positive semidefinite $|X|=\sqrt{X X^{*}}$ where $\lambda_{i}(X)=s_{i}(X)$ for all i. Then the Matrix sum inequality qwith respect to the singular values is $\sum_{i=1}^{n} s_{i}(A+B) \leq \sum_{i=1}^{n} s_{i}(A)+\sum_{i=1}^{n} s_{i}(B)$

B. Adjacency Matrix of a Semigraph

Consider a semigraph $\mathrm{SG}(\mathrm{V}, \mathrm{X})$. Let $V=\{1,2, \ldots . p\}$ be vertex set and $X=\left\{e_{1}, e_{2}, \ldots e_{q}\right\}$, the edge set where $e_{j}=\left(i_{1}, i_{2}, \ldots . . i_{j}\right)$ and $i_{1} i_{2}, \ldots \ldots i_{j}$ are distinct elements of $\mathrm{V}$, then the $p \times p$ matrix $\mathrm{A}$ is the Adjacency matrix of semigraph $S G(V, X)$ whose entries are given by $a_{i j}=$ cardinality of fp edge $\left(v_{i}, v_{j}\right)-1$, if $v_{i}$ and $v_{j}$ are adjacent

$$
=0, \text { otherwise. }
$$




\section{Change in Semigraph Energy Due to Edge Deletion and its Relation with Distance Energy}

\section{C.Energy of a graph}

Consider the graph $G$ not directed, not infinite and not multiple graph with number of vertices $n$ and number of edges $\mathrm{m}$. Consider the adjacency matrix $A=\left(a_{i j}\right)$ of graph $G$, then the eigenvalues assumed in non-increasing order $\lambda_{1}, \lambda_{2}, \ldots . \lambda_{n}$ of $A(G)$, are the graph eigenvalues. Then $E(G)$ graph energy $G$, is defined as $E(G)=$ $\sum_{i=1}^{n}\left|\lambda_{i}\right|$. The spectrum $\mathrm{G}$ is the set $\left\{\lambda_{1}, \lambda_{2}, \ldots . \lambda_{n}\right\}$ denoted by $\operatorname{Spec} G$. If $\mathrm{G}$ has distinct eigenvalues say, $\lambda_{1}>\lambda_{2}>\ldots .>\lambda_{n}$ and if their multiplicities are $m\left(\lambda_{i}\right)$ then

$\operatorname{Spec} G=\left(\begin{array}{ccccc}\lambda_{1} & \lambda_{2} & . . & . . & \lambda_{n} \\ m\left(\lambda_{1}\right) & m\left(\lambda_{2}\right) & . . & . . & m\left(\lambda_{n}\right)\end{array}\right)$

The spectrum of the graph does not depend on the labeling of the vertex set of the graph. Since we have the matrix symmetric and real with the trace zero, we have sum of the real real eignvalues to be zero.

\section{A kind of energy with respect to the distance in a semigraph}

If $S G$ is a simple connected semigraph and the vertices are labelled as $v_{1}, v_{2}, \ldots v_{n}$. then the matrix of a semigraph with respect to the distance, $S G$ is given by a square matrix $D(G)=\left(d_{i j}\right)$ in which the entries are the distance between the vertices $v_{i}$ and $v_{j}$ in $S G$. The eigenvalues of the matrix we consider $\mu_{1}, \mu_{2}, \ldots \mu_{n}$ are said to be the distance eigenvalues. As we have a matrix which is symmetric, we have real eigenvalues in order $\mu_{1} \geq \mu_{2} \geq \ldots \geq \mu_{n}$. The energy with respect to distance of a semigraph $E_{D}(S G)$ is defined as

$$
E_{D}(S G)=\sum_{i=1}^{n}\left|\mu_{i}\right| \text {. }
$$

\section{E.Energy of a Semigraph}

Let $S G$ be not directed, not infinite and not a multiple semigraph with number of vertices $n$ and number of edges $m$. Consider $A=\left(a_{i j}\right)$ the adjacency matrix of semigraph $S G$. The eigenvalues $\eta_{1}, \eta_{2}, \ldots . . \eta_{n}$ of $A(S G)$, taken not increasing order, are the eigenvalues of the semigraph $S G$. Energy of a semigraph $S G$, denoted by $E(S G)$ is defined as $E(S G)=\sum_{i=1}^{n}\left|\eta_{i}\right|$. The set $\eta_{1}, \eta_{2}, \ldots . \eta_{n}$ is the spectrum of $S G$ and is denoted by Spec $S G$. If the eigenvalues of $S G$ are distinct say, $\eta_{1}>\eta_{2}>\ldots .>\eta_{n}$ with their multiples $m(\eta i)$ then we write
$\operatorname{Spec} S G=\left(\begin{array}{ccccc}\eta_{1} & \eta_{2} & . . & . . & \eta_{n} \\ m\left(\eta_{1}\right) & m\left(\eta_{2}\right) & . . & . . & m\left(\eta_{n}\right)\end{array}\right)$

The spectrum of the above graph does not depend on the labeling of the vertex set of the graph. Since we have the matrix symmetric and real with the trace zero, we have sum of the real real eignvalues to be zero.

\section{F.Theorem 3.4}

If $S H$ is a non-empty induced subsemigraph of a simple connected regular semigraph $S G$, then $E(S G)-E(S H) \leq E\left(S G^{\prime}\right) \leq E(S G)+E\left(S H^{\prime}\right)$

\section{Proof}

$S G$ is a connected simple semigraph. $S H$ be an induced subsemigraph of $S G$, containing all edges of $S G$ connecting two vertices of $S H$ Let $S G-S H$ be the semigraph, having got from $S G$ removing all vertices of $\mathrm{SH}$ and the edges incident with $\mathrm{SH}$. If there are two semigraphs $S G 1$ and $S G 2$ semigraphs with out any vertices in common and if we consider $S G 1 \oplus S G 2$ as the semigraph with vertex set and the edge set $V(S G 1) \cup V(S G 2) ; E(S G 1) \cup E(S G 2)$ respectively. Hence

$A(S G 1 \oplus S G 2)=A(S G 1) \oplus A(S G 2)$

$$
\begin{aligned}
& A(S G)=\left(\begin{array}{cc}
A(S H) & X^{T} \\
X & A(S G-S H)
\end{array}\right) \\
& =\left(\begin{array}{cc}
A(S H) & 0 \\
0 & 0
\end{array}\right)+\left(\begin{array}{cc}
0 & X^{T} \\
X & 0
\end{array}\right)
\end{aligned}
$$

In which the edges joining $S H$ and $S G-S H$ is $X$.

Also if $A\left(S G^{\prime}\right)=\left(\begin{array}{cc}0 & X^{T} \\ X & 0\end{array}\right)$

Using the inequality theorem of matrices with respect to singular value

$E(S G) \leq E(S H)+E\left(S G^{\prime}\right)$, which gives one part of the inequality

$$
\begin{aligned}
& E(S G)-E(S H) \leq E\left(S G^{\prime}\right) \\
& A\left(S G^{\prime}\right)=A(S G)+\left(\begin{array}{cc}
-A(S H) & 0 \\
0 & 0
\end{array}\right)
\end{aligned}
$$

By the inequality theorem with respect to singular values,

$E\left(S G^{\prime}\right) \leq E(S G)+E(S H)$

$E\left(S G^{\prime}\right) \leq E(S G)+E(S H)---i i$

From $i$ and $i i$ it follows

$E(S G)-E(S H) \leq E\left(S G^{\prime}\right) \leq E(S G)+E(S H)$

Both the left and right equality holds when $E(S H)=\phi$ 
G.Theorem 3.5 If $S H$ is a non-empty induced subsemigraph of a simple connected semigraph $S G$. Then

$$
\begin{gathered}
E_{D}(S G)-E_{D}(S H) \leq E\left(S G^{\prime}\right)< \\
E_{D}(S G)+E_{D}(S H){ }^{. \text {Proof }}
\end{gathered}
$$

$S G$ is a connected simple semigraph. $S H$ be an induced subsemigraph of $S G$,containing all edges of $S G$ joining two vertices of $S H$ Let $S G-S H$ denote the semigraph, having got from $S G$ removing all vertices of $S H$ and the edges that are incident with $S H$. If $S G 1$ and $S G 2$ are two semigraphs not having any vertices in common, and if we consider $S G 1 \oplus S G 2$ the semigraph with vertex set and the edge set $\quad V(S G 1) \cup V(S G 2)$

$E(S G 1) \cup E(S G 2)$ respectively. Hence

$A(S G 1 \oplus S G 2)=A(S G 1) \oplus A(S G 2)$

$D(S G 1 \oplus S G 2)=D(S G 1) \oplus D(S G 2)$

$$
\begin{array}{r}
D(S G)=\left(\begin{array}{cc}
D(S H) & X^{T} \\
X & D(S G-S H)
\end{array}\right) \\
=\left(\begin{array}{cc}
D(S H) & 0 \\
0 & 0
\end{array}\right)+ \\
\left(\begin{array}{cc}
0 & X^{T} \\
X & D(S G-S H)
\end{array}\right)
\end{array}
$$

$$
\begin{aligned}
& \text { From } \quad \begin{array}{l}
i \\
E_{D}(S G)-E_{D}(S H)
\end{array} \\
& \\
& \leq E\left(S G^{\prime}\right) \\
& \leq E_{D}(S G)+E_{D}(S H)
\end{aligned}
$$

Both the left and right equality holds when $E_{D}(S H)=\phi$

\section{CONCLUSION}

We find that energy of semigraphs increases or decreases or remains the same due to edge deletion. We can study the semigraph energy change due to vertex deletion using singular value inequality. We can study its relation with other forms of energies of semigraphs due to edge deletion and vertex deletion

\section{REFERENCES}

[1] AIM Workshop on spectra of families of matrices described by graphs, digraphs and sign patterns: open questions, December 7, 2006.

[2] Bhatia.R, Matrix Analysis, Springer, New York, 1996.

[3] Balakrishnan.R, The energy of a graph Linear Algebra and its Applications Vol 387 287-295, 2004.

[4] Balakrishnan.R, The skew energy of a digraph. Linear Algebra and its Applications, 2009

[5] Cheng.C.M, Horn.R.A and Li.C.K, Inequalities and equalities for the cartesian decomposition of complex matrices. Linear Algebra Appl., 341; 219-237, 2002.

[6] C.M.Deshpande and Y S Gaidhani, About Adjacency Matrix of Semigraphs, International Journal of Applied Physics and Mathematics, Vol 2, No. 4, July 2012.

[7] Gutman.I, The energy of a graph. Ber.Math-Statist.Sekt.Forsch.Graz, 103:1-22, 1978

[8] Hosoya.H, Murakami, Gotoh.M,Distance polynomial and characterization of a graph, Natur.Sci.Rept.Ochanumizu Univ.24(1973), 27-34.

[9] Hua.H, On minimal energy of unicyclic graphs with prescribed girth and pendant vertices, MATCHCommun.Math.Comput.Chem.57(2007), 351-361.

[10] Horn.R and Johnson.C, Matrix Analysis. Cambridge University Press, Cambridge, 1989.

[11] Indulal.G,Gutman.I,Vijaykumar, On the distance energy of a graph, MATCH Commun.Math.Comput.Chem.60(2008), 461-472.

$X$ represents edges connecting $S H$ and $S G-S H$.

$$
\begin{aligned}
& D(S G)=\left(\begin{array}{cc}
D(S H) & 0 \\
0 & 0
\end{array}\right)+ \\
& \left(\begin{array}{cc}
0 & X^{T} \\
X & A(S G-S H)
\end{array}\right) \\
& \text { Also if } A\left(S G^{\prime}\right)=\left(\begin{array}{cc}
0 & X^{T} \\
X & A(S G-S H)
\end{array}\right) \\
& D(S G)=\left(\begin{array}{cc}
D(S H) & 0 \\
0 & 0
\end{array}\right)+A\left(S G^{\prime}\right)
\end{aligned}
$$

[12] Jane Day and Wasin So, Graph Energy Change due to Edge Deletion October 12, 2007-AIM Workshop on spectra of families of matrices described by graphs, digraphs and sign patterns: open questions, December 7, 2006.

[13] Jane Day and Wasin So, Singular value inequality and graph energy change, Electronic Journal of Linear Algebra, Volume 16, 291-299, September 2007.

[14] Murugesan.N, Meenakshi.K, Singular value inequality and relation between energy and distance energy, IEEE proceedings, April 2010.

[15] Ramane.H.S, Revankar.Deepak.S, Ivan Gutman, Siddani Bhaskara Rao, Devadas Acharya.B and Walikar.H.B, Bounds for the distance energy of a graph, Kragujevac J.Math. 31 (2008) 59-68.

[16] Sampathkumar.E, Semigraphs and their Applications Report on the DST project submitted to DST, India, May 2000.

[17] Ahmed, Syed Thouheed, M. Sandhya, and Sharmila Sankar. "A Dynamic MooM Dataset Processing Under TelMED Protocol Design for QoS Improvisation of Telemedicine Environment." Journal of medical systems 43, no. 8 (2019): 257.

[18] Ahmed, Syed Thouheed, M. Sandhya, and Sharmila Sankar. "An Optimized RTSRV Machine Learning Algorithm for Biomedical Signal Transmission and Regeneration for Telemedicine Environment." Procedia Computer Science 152 (2019): 140-149.

[19] Patil, Kiran Kumari, and Syed Thouheed Ahmed. "Digital telemammography services for rural India, software components and design protocol." In 2014 International Conference on Advances in Electronics Computers and Communications, pp. 1-5. IEEE, 2014.

[20] Thouheed, Syed, S. Ahmed, M. Sandhya, and S. Shankar. "ICT's Role in Building and Understanding Indian Telemedicine Environment: A Study." In Information and Communication Technology for Competitive Strategies. Lecture Notes in Networks and Systems, vol 40. Springer Singapore, 2019.

By singular value inequality theorem,

$$
\begin{aligned}
& E\left(S G^{\prime}\right) \leq E_{D}(S G)+E_{D}(S H) \\
& E\left(S G^{\prime}\right) \leq E_{D}(S G)+E_{D}(S H)---i i
\end{aligned}
$$

